\title{
Electrochemical characteristics of bioresorbable binary MgCa alloys in Ringer's solution: Revealing the impact of local pH distributions during in-vitro dissolution
}

\author{
D. Mareci ${ }^{1}$, G. Bolat ${ }^{1}$, J. Izquierdo ${ }^{2}$, C. Crimu ${ }^{3}$, C. Munteanu ${ }^{3}$, I. Antoniac ${ }^{4}$, R.M. Souto ${ }^{2}$ \\ ${ }^{1}$ Faculty of Chemical Engineering and Environmental Protection, The “Gheorghe Asachi” Technical \\ University of Iasi, 700050, Iasi, Romania \\ ${ }^{2}$ Department of Chemistry, University of La Laguna, P.O. Box 456, E-38200 La Laguna (Tenerife), \\ Spain \\ ${ }^{3}$ Faculty of Mechanical Engineering, The “Gheorghe Asachi” Technical University of Iasi, 700050, Iasi, \\ Romania \\ ${ }^{4}$ Faculty of Materials Science and Engineering, Politehnica of Bucharest, 060042, Bucharest, Romania
}

\begin{abstract}
Biodegradable magnesium-calcium (MgCa) alloy is a very attractive biomaterial. Two MgCa alloys below the solid solubility of Ca were considered, as to solely investigate the effect of Ca content on the behaviour of magnesium and the $\mathrm{pH}$ changes associated to metal dissolution. X-ray diffraction analysis and optical microscopy showed that both Mg-0.63Ca and Mg-0.89Ca alloys were solely composed of $\alpha(\mathrm{Mg})$ phase. Degradation characteristics and electrochemical characterization of MgCa alloys were investigated during exposure to Ringer's solution at $37^{\circ} \mathrm{C}$ by electrochemical impedance spectroscopy and scanning electrochemical microscopy. The impedance behaviour showed both capacitive and inductive features that are related to the alloy charge transfer reaction and the relaxation of the absorbed corrosion compounds, and can be described in terms of an equivalent circuit. Scanning electron microscopy (SEM) was employed to view the surface morphology of the MgCa samples after 1 week immersion in Ringer's solution showing extensive precipitation of corrosion products, whereas the substrate shows evidence of a non-uniform corrosion processes. Energy dispersive analysis showed that the precipitates contained oxygen, calcium, magnesium and chlorine, and the Mg:Ca ratios were smaller than in the alloys. Scanning electrochemical microscopy (SECM) was used to visualize local pH changes associated to these physicochemical processes with high spatial resolution. The occurrence of $\mathrm{pH}$ variations in excess of 3 units between anodic and cathodic half-cell reactions was monitored in situ.
\end{abstract}

Keywords: MgCa alloys; corrosion resistance; electrochemical characterization; EIS; SECM; XRD; SEM; local pH distributions. 


\section{Introduction}

Metallic implants placed in the human body suffer a major challenge from stress shielding that reduces their service life eventually requiring surgical replacement. Stress shielding is the inhomogeneous transfer of stress between the implant and the bone [1,2], and it can cause bone resorption, cell death and implant loosening [3]. Therefore, intensive efforts are directed in recent years to the development of "biodegradable implants". These are non-toxic implant materials that become resorbed by the human body after a certain period of time. $\mathrm{Mg}$ and its alloys appear to be the best candidates for the production of biodegradable metallic implants due to their biocompatibility and excellent mechanical properties [4-8]. Indeed, a substantial amount of $\mathrm{Mg}$ is taken into the human body daily and is beneficial for bone strength and growth [4], and stimulates the metabolism as cofactor for certain enzymes [9]. On the other hand, the fast production of metallic ions from magnesium implants can be excreted in the urine without adverse reactions [10]. But the high degradation rates of Mg alloys can hardly meet the requirements for biomedical applications because the biodegradability process is usually faster than the time required to heal the bone [11,12]. In addition, hydrogen gas is quickly generated in the corrosion process and can originate adverse reactions in the tissues adjacent to the implant [12,13]. As result, implants made of pure magnesium or magnesium alloys are still not commercially available $[13,14]$.

The high corrosion rates of pure magnesium can be effectively slowed down by alloying the metal with other elements [15]. Currently, magnesium alloys are extensively investigated, and various magnesium alloys containing aluminium have already found application in the fabrication of lightweight materials for the automotive and aerospace transportation industries [16]. It was thus rather natural that many of these alloys, as such or containing small calcium additions [17], were investigated regarding their degradation characteristics in simulated physiological environments $[4,12,18]$. Nevertheless, those engineering alloys, even containing small calcium additions, should not be considered for implant application because they usually contain aluminium as alloying element. This element has been associated to damage in muscle fibers [19] and Alzheimer disease [19,20] .

Therefore, other alloying alternatives are explored, including rare-earth elements [13,21], zinc [22-26], calcium [27,28], manganese [26,29], zirconium [30], and more recently strontium [30-32]. Among them, the best cytocompatibility is shown by calcium [12], whereas the other elements are often

investigated as minor constituents in Mg-Ca based alloys [12,25,26] due to their effects on the mechanical properties and the corrosion resistance of the resulting material [26]. Calcium has been 
selected as an alloying element considering that the degradation products are expected to be non-toxic $[33,34]$ since calcium is a major component in human bone and is essential in chemical signalling with cells [35]. Moreover, magnesium is necessary for the calcium incorporation into the bone [27]. Therefore, MgCa alloys constitute a promising alternative as biodegradable implant materials because they also have the potential to minimize stress shielding whereas providing good biocompatibility and adequate strength [10]. The recent development of $\mathrm{Mg}-1 \mathrm{Ca}$ alloy revealed that it had the acceptable biocompatibility to be regarded a new biodegradable bone implant material [26]. Unfortunately, with increasing $\mathrm{Ca}$ content, more $\mathrm{Mg}_{2} \mathrm{Ca}$ phase precipitates along grain boundaries, weakening both the mechanical properties and the corrosion resistance of the MgCa alloy [36].

Electrochemical characterization of the corrosion of Mg-based materials is commonly performed by the application of DC electrochemical methods, but big differences are often reported due to the high reactivity of these materials in aqueous environments. In this context, Shi and coworkers [37] recommended those measurements to be complemented by at least two other experimental methods, such as weight loss rate, hydrogen evolution rate, or/and rate of $\mathrm{Mg}^{2+}$ leaving the metal surface. Unfortunately, the spontaneous degradation reactions experienced by magnesium-based materials are heterogeneously distributed over their surface [38,39], whereas those experimental techniques can only average the behaviour of the whole exposed areas, and therefore do not provide sensitive monitoring of the actual physicochemical processes involved. For instance, local $\mathrm{pH}$ changes occur as a result of the surface electrochemical reactions on spontaneously corroding materials in aqueous environments, though they pass unnoticed when employing conventional electrochemical techniques for their characterization because they average the behaviour of the complete exposed surface. Cathodic processes lead to strong alkalization, whereas anodic dissolution may result in hydrolysis and eventual acidification [40]. Unfortunately, the onset of these local $\mathrm{pH}$ distributions has been ignored during the electrochemical characterization of MgCa alloys until now, but they may exert a major impact on the biocompatibility of these alloys. Although the $\mathrm{pH}$ of the intercellular fluid remains neutral and practically unchanged in typical physiological conditions [12], it may greatly vary either as result of infection or during surgical procedures, effectively modifying the aggressivity of the environment associated to the inflammatory reactions. Indeed, $\mathrm{pH}$ changes can promote the activation of the inflammatory response by themselves.

With the development of the scanning microelectrochemical microscope (SECM), in vitro studies of the dissolution and passivation reactions of biomaterials can be performed with high spatial 
and chemical resolution [41-45]. The basic operation principle of the technique consists in the transformation of a redox-active species to another redox state at a microelectrode tip while scanning a surface in close proximity. The amperometric current measured at the tip -usually a noble metal such as platinum or a carbon fibre- is influenced by both the surface reactivity of the investigated substrate and the distance between its surface and the tip. That is, it senses conductivity at the substrate/electrolyte interface and in the thin layer electrolyte, leading to the measurement of surface reactivity distributions. Recently, antimony microelectrodes have been implemented in SECM to detect local pH changes resulting from corrosion processes in magnesium and magnesium alloys [46]. These $\mathrm{pH}$ variations may significantly alter the physiological media and proteins functionality, and must be monitored with high spatial resolution, because electrochemical microcells formed at the metal surfaces may lead to the establishment of a highly localized active site. Furthermore, antimony electrodes present the advantage of behaving as dual-function amperometric/potentiometric sensors, so the proximity of the substrate during approach operation can be accurately controlled from its amperometric response in SECM, either in conventional [40] or alternating current modes [46].

In the present study, the degradation characteristics of MgCa alloys as biodegradable biomaterials for biomedical applications were evaluated using electrochemical impedance spectroscopy (EIS) and scanning electrochemical microscopy (SECM). The alternating current (AC) impedance method is particularly useful when monitoring electrochemical change as a function of time, and thus it is especially suited for the characterization of the corrosion resistance of metals and alloys. Using electrochemical impedance spectroscopy much more information can be collected than by direct current (DC) electrochemical methods. Next, the scanning microelectrochemical method allowed detection of the active sites on the corroding material at which alkalization or acidification occurred, in order to establish the range of the $\mathrm{pH}$ changes related to magnesium degradation.

\section{Materials and methods}

\subsection{Materials}

Two MgCa alloys were obtained by melting at $1800^{\circ} \mathrm{C}$, under controlled atmosphere of $\mathrm{Ar}$, in a high frequency induction furnace $(20 \mathrm{KHz})$ type Enterprise UltraCast (Inductronix, Anaheim CA, USA). The nominal chemical compositions (wt.\%) of the experimental alloys were: Mg-0.63Ca (subsequently named Mg0.6Ca), and Mg-0.89Ca (named Mg0.9Ca). The samples were subsequently high pressure torsion (HPT) deformed using an applied load of ca. 5 GPa under 0.2 rpm rotation speed. 
The actual chemical compositions were determined by EDX analysis using a scanning electron microscope (Quanta 3D, FEI, Hillsboro, OR, USA) equipped with an EDX detector and they are given in Figure 1. The percentages given in the Figure were calculated as the average of ten values.

Electrochemical tests were performed in Ringer's physiological solution of composition: $8.6 \mathrm{~g} / \mathrm{L}$ $\mathrm{NaCl}, 0.3 \mathrm{~g} / \mathrm{L} \mathrm{KCl}$, and $0.48 \mathrm{~g} / \mathrm{L} \mathrm{CaCl}_{2}$. The solution was produced using analytical grade chemicals and high purity deionized water.

\subsection{Alloy characterization methods}

The microstructure of MgCa alloys was examined using a metallographic microscope (DMI5000 M, Leica, Wetzlar, Germany) equipped with a dedicated digital camera connected to a personal computer. Image analysis was performed using the Leica Application Suite software program. Prior to optical imaging, the samples were first mechanically abraded using a sequence of silicon carbide abrasive papers, followed by a final polishing step using $0.3 \mu \mathrm{m}$ alumina suspension. Next, the samples were cleaned in alcohol and distilled water using an ultrasonic bath, and finally etched in a solution containing $12 \mathrm{~g}$ picric acid, $80 \mathrm{~mL}$ acetic acid, $350 \mathrm{~mL}$ ethanol, and $80 \mathrm{~mL}$ water.

$\mathrm{X}$-ray diffraction (XRD) was employed for the identification the phase structure. XRD spectra were recorded using an X'Pert PRO MRD (PANalytical, Almelo, The Netherlands) diffractometer equipped with a $\mathrm{Cu} \mathrm{K}_{\alpha}$ anode, and the XRD patterns were scanned in the $30^{\circ} \leq 2 \theta \leq 80^{\circ}$ range.

Wettability of the surface preparations of the MgCa alloys was established by contact angle measurements. They were performed using an EasyDrop instrument (Kruss, Germany) controlled by a personal computer and specific software (Drop Shape Analysis, Kruss, Germany).

\subsection{EIS analysis}

The test MgCa alloys were placed in a glass corrosion flow cell kit (C145/170, Radiometer Analytical, Lyon, France), which was filled with both Ringer's solution. A saturated calomel electrode was used as the reference electrode, and a platinum coil as the counter electrode. The potentials in this paper are reported versus the saturated calomel electrode (SCE). The temperature of the electrochemical cell was maintained at $37 \pm 1^{\circ} \mathrm{C}$.

Electrochemical measurements were performed using a potentiostat model PARSTAT 4000 (Princeton Applied Research, Oak Ridge, TN, USA). The instrument was controlled by a personal computer and VersaStudio software. Prior to testing, the working electrodes were mechanically abraded 
using emery paper up to 2000 grit, next polished with $0.3 \mu \mathrm{m}$ alumina suspension, ultrasonically cleaned in acetone and deionized water, and finally dried in open air.

Electrochemical impedance spectra were measured over a frequency range extending from $10^{5}$ $\mathrm{Hz}$ to $10^{-3} \mathrm{~Hz}$ using a $10 \mathrm{mV}$ amplitude $\mathrm{AC}$ voltage signal. The impedance data recorded in the frequency interval comprised between $10^{-2} \mathrm{~Hz}$ and $10^{-3} \mathrm{~Hz}$ exhibited large dispersion, and they were discarded. The EIS tests were recorded at the open circuit potential developed by the alloys after various immersion periods in the test solution, namely 1 hour, 1 day, and 1 week. Analysis of the spectra was performed by equivalent circuit (EC) fitting using ZSimpWin software.

\subsection{SECM operation}

The fabrication of the pH-sensitive dual function antimony electrodes has been described elsewhere [47]. In brief, antimony powder is melted under flame and inserted by suction in the lumen of a glass capillary, which is afterwards pulled until the desired micrometer-range diameter is reached. Then, the as fabricated microcapillaries are glued inside a ca. $1 \mathrm{~mm}$ inner diameter capillary, leaving a 2-3 mm length of the antimony-glass microcapillary to reach out from the lumen of the thicker capillary. Epoxy glue is used to avoid entrance of electrolyte to the lumen of the latter. Electric connection is then ensured with either liquid mercury or soldering material in contact with a copper wire. Antimony electrodes herein employed had a nominal diameter of ca. $30 \mu \mathrm{m}$ as antimony microdisk active surface. Open circuit potential responses of the electrodes were finally calibrated as a function of $\mathrm{pH}$.

The electrochemical cell used for the SECM characterization, purchased from Sensolytics (Bochum, Germany) consisted in a coated-metal base with internal flow channels. A $1.5 \mathrm{~cm}$ diameter sample target was placed over this piece, and fixed with a methacrylate adapted piece and a $1 \mathrm{~cm}$ o-ring to prevent leakage. In this manner, cell allowed heated up water to flow through its internal channels, so that the temperature of the sample, in contact with the coated-metal based, could be maintained $37^{\circ} \mathrm{C}$. The antimony microelectrode, an $\mathrm{Ag} / \mathrm{AgCl} / 3 \mathrm{M} \mathrm{KCl}$ reference electrode and a platinum counterelectrode were placed in solution to complete the three-electrode configuration cell.

Electrodes were connected to an SECM equipment purchased from Sensolytics (Bochum, Germany), in association with external PARSTAT 386 potentiostat and 5210 Lock-In amplifier supplied by Princeton Applied Research (Oak Ridge, TN, USA). The signals measured by the external instruments could be processed with the Sensolytics software through analogic channels, and accurately correlated with the probe position. First, this configuration was used to apply a sinusoidal $\pm 10 \mathrm{mV}$ 
potential perturbation to the antimony electrode superimposed to its spontaneous OCP in bulk solution, and subsequent alternating current was monitored as a function of the tip displacement towards the surface (computer controlled). When the AC magnitude suddenly decayed, tip movement was stopped and it was retracted $10 \mu \mathrm{m}$ away, in a way that the mean distance while scanning was around 20-30 $\mu \mathrm{m}$. Video camera assistance was used to confirm that the tip was placed at this approximate distance. Then, voltage perturbation was switched off and open circuit potential of the antimony electrode was recorded instead. For that purpose, a voltage follower was included in the measuring circuit containing a $10^{12} \Omega$ input impedance operational amplifier, and potential was measured as a function of tip position while moving from active surface to bulk solution (vertical profiles), and in parallel with respect to the metal surface (2-D scans). The following scan rates were used: $5 \mu \mathrm{m} \mathrm{s}^{-1}$ for the vertical approach scan lines, and $10 \mu \mathrm{m} \mathrm{s}^{-1}$ for the scans parallel to the surface.

\subsection{SEM characterization of the corroded surfaces}

The surface morphology of the two MgCa alloys after 1-week exposure times to the Ringer's solution, both before and after removal of the corrosion products deposited on their surface, was assessed using scanning electron microscopy (SEM, Quanta 3D, FEI, Hillsboro, OR, USA) equipped with an EDX detector. The corrosion products were removed from the materials using a chromic acid solution, according to ASTM G1-90 [48].

\section{Results and discussion}

\subsection{Structural analysis}

An optical micrograph showing the microstructure of the Mg0.9Ca alloy is given in Figure 2. The microstructure of this alloy is characterized by equiaxed $\alpha(\mathrm{Mg})$ grain morphology. The X-ray diffractometry patterns depicted in Figure 3 confirm the two MgCa alloys considered in this work are constituted by a single-phase, namely $\alpha(\mathrm{Mg})$ solid solution.

Figure 4 shows the contact angle measurement performed on the Mg0.9Ca alloy after 1 week immersion time in Ringer’s solution. Both Mg0.9Ca and Mg0.6Ca (not shown) exhibited water contact angles $(\theta)$ close to $90^{\circ}$, and are thus defined as hydrophobic surfaces. Therefore, a hydrophobic behaviour similar to pure magnesium is exhibited by these MgCa alloys, since it has been reported that the contact angle for the metal is $87 \pm 1^{\circ}$ [49]. 


\subsection{Electrochemical impedance spectroscopy}

The electrochemical characteristics of the MgCa alloys exposed to naturally-aerated Ringer's solution at $37^{\circ} \mathrm{C}$ were monitored using electrochemical impedance spectroscopy (EIS) over the $10^{5}-$ $10^{-2} \mathrm{~Hz}$ frequency range. The AC potential signal was imposed around their open circuit potential $\left(E_{\text {cor }}\right)$ values. The impedance spectra measured at three different immersion times are displayed in Figure 5 in the form of Nyquist (imaginary $\left(Z_{\mathrm{im}}\right)$ versus real $\left(Z_{\text {re }}\right)$ components of the impedance) and Bode (impedance modulus $|Z|$ and phase angle $(-\Phi)$ versus frequency ( $f$ ) diagrams).

The Nyquist plots of the alloys show one capacitive arc in the high and intermediate frequency ranges, followed by an inductive arc in the low frequency range for all the three immersion times (cf. Fig. 5A and 5C for Mg0.6Ca and Mg0.9Ca, respectively). The capacitive arc is associated to the dielectric properties of the corrosion products layer formed on the alloy surface. In this case, the inductive arc is associated to metallic dissolution processes. The size of the impedance plots was observed to decrease steadily for longer exposures, the biggest diagram being displayed by the spectra determined after 1 hour immersion for both MgCa alloys. This finding evidences that the corrosion resistance of the materials deteriorated in Ringer's solution with the elapse of time. Bode spectra recorded at open circuit potential, with the MgCa alloys for a different period of time in Ringer's solution, at $37^{\circ} \mathrm{C}$, are shown in Figure 6(A-B). The corresponding Bode-phase diagrams exhibit one relaxation time constant in the medium and high frequency ranges, i.e., only one peak can be found in the plots given in Figs. 5B and 5D. Conversely, smaller impedance values were measured in the low frequency range, associated to the observation of an inductive behaviour for the system. However, as a general rule, the impedance values determined at any frequency value after 1 hour immersion in Ringer's solution are larger than those found at longer exposures, a trend that is even more pronounced in the spectra measured after 1 week. This behaviour can be attributed to the formation of relatively compact corrosion products layer in the Ringer's solution.

Analysis of the impedance spectra in terms of an equivalent circuit (EC) allowed the values of the impedance parameters to be extracted. The Bode diagrams were satisfactorily simulated using the model presented in Fig. 6 as indicated between the good agreement between the calculated (solid line) and the measured (symbols) data over the complete frequency range. In fact, the variability of the fitted impedance parameters was smaller than $5 \%$ in all cases. Table 1 lists the results of those fits. Constant

phase elements (CPE) were used instead of pure capacitances because of the non-ideal capacitive 
response due to the distributed relaxation feature of the surface films present on the alloys. The impedance representation of a CPE is given by:

$$
Z_{\mathrm{CPE}}=\frac{1}{Y_{0}(j \omega)^{\mathrm{n}}}
$$

where $\omega$ is the angular frequency, $Y_{0}$ is a constant, and the value of the exponent $n$ indicates the deviation from the ideal capacitive behaviour (corresponding to $n=1$ ).

The following impedance elements were considered. $R_{\text {sol }}$ is the resistance of solution occurring between the sample and the reference electrode. This parameter has a value around $70 \pm 2 \Omega \mathrm{cm}^{2}$ in Ringer's solution, and is not listed in Table 1 . The elements $R_{1}$ and $Q_{1}$ describe the resistance and the constant phase elements of the corrosion products layers, and they are determined in the high and medium frequency ranges. The occurrence of the inductive combination $R_{1} L$ is attributed to the relaxation of the corrosion products formed on both $\mathrm{MgCa}$ alloys. The characteristic corrosion resistances of the two MgCa alloys can be compared based on their values for $R_{1}$. For a given alloy the values of $R_{1}$ decrease with increasing the immersion time. These results indicate the failure of the surface layer formed by the precipitation of corrosion products. However, it can be deduced that the impedance of the MgCa alloy increased when the material contained higher Ca contents.

The oxide films formed in aqueous electrolytes mainly consist of $\mathrm{Mg}(\mathrm{OH})_{2}$ which retards the corrosion process [15]. The formation of the $\mathrm{Mg}(\mathrm{OH})_{2}$ layer is accompanied by vigorous hydrogen evolution as directly observed by visual inspection [50]. As a result, the formed $\mathrm{Mg}(\mathrm{OH})_{2}$ layer cannot provide effective protection to the $\mathrm{Mg}$ substrate. Moreover, $\mathrm{Mg}(\mathrm{OH})_{2}$ layer undergoes severe attack in the presence of chloride anions leading to the release of soluble compounds [51]. This dissolution stage is preceded by the adsorption of chlorides on the hydroxide layer given by:

$$
\begin{gathered}
\mathrm{Mg}(\mathrm{OH})_{2}+\mathrm{Cl}^{-} \rightarrow \mathrm{MgOHCl}_{\mathrm{ad}}+\mathrm{OH}^{-} \\
\mathrm{MgOHCl}_{\mathrm{ad}} \rightarrow \mathrm{Mg}^{2+}+\mathrm{OH}^{-}+\mathrm{Cl}^{-}
\end{gathered}
$$

\subsection{SEM analysis of the corroded surfaces}

The surface topography of the MgCa alloys was examined by scanning electron microscopy (SEM). Figure 7 shows the micrographs taken from retrieved samples after 1 week immersion time in naturally aerated Ringer's solution at $37^{\circ} \mathrm{C}$. The samples were left effectively unpolarized in the test solution at their corresponding $E_{\text {cor }}$ values since the EIS measurements only required a minute potential perturbation during spectra acquisition. Extensive corrosion damage is found on the alloys, which are 
covered by relatively massive corrosion products containing rather large clusters of crystallites. Additionally, some holes can also be observed in their surface. The occurrence of oxygen, calcium, magnesium and chlorine was identified from the EDX spectra measured at 5 different spots on the surface leading to the average values displayed in Figure 8. A significant decrease of the relative percentage of $\mathrm{Mg}$ is encountered, likely due to the dissolution process that preferentially affects to this element. Furthermore, EDX analysis proved the existence of chlorides in the corrosion product layer deposited on the surface of the materials.

The morphology of the underlying corroded metals was also characterized using scanning electron microscopy. In this case, the layers of corrosion products were removed through their dissolution in a chromic acid solution (200 g/L $\mathrm{CrO}_{3}$ and $10 \mathrm{~g} / \mathrm{L} \mathrm{AgNO}_{3}$ ). The SEM images of the bare surfaces are shown in Figure 9. As result of the exposure of the MgCa alloys to Ringer's solution for 1 week, the metallic substrate suffered extensive damage over the complete exposed surface, yet exhibiting characteristics of a localized corrosion attack. In fact, some Mg alloys usually suffer from non-uniform (localized) corrosion attack [16,52]. The EDX spectra given in Figure 10 indicate the absence of chlorides on the underlying corroded surface of the MgCa alloys, thus they are exclusively associated to the precipitated corrosion products.

\subsection{SECM characterization}

Magnesium-based materials are expected to promote electrolyte alkalization upon corrosion processes [39], due to the combination of hydrogen ions reduction in the anodic process, and the formation of hydroxide ions in the cathodic half-reaction, respectively given by equations (2) and (3). These processes may be highly heterogeneous and eventually result in greater local effects than those predicted using conventional electrochemical methods, because the latter average the response of the complete exposed material. The SECM technique has been employed to characterize the $\mathrm{pH}$ variations occurring during the vigorous degradation of these materials in Ringer's physiological solution with high spatial resolution.

A selection of vertical $\mathrm{pH}$ profiles (i.e., z-approach curves), determined at various immersion times using the pH-sensitive antimony electrode, are plotted in Figure 11. Alkalization of the electrolyte volume adjacent to the material surface is found in all measurements, though it occurs in a greater extent over the Mg0.9Ca alloy. Conversely, some measurements show the occurrence of mixed response behaviours when the sensor is placed in close proximity to the Mg0.6Ca surface. That is, though the 
electrolyte undergoes steady alkalization as result of the degradation reactions of the material, yet slightly less alkaline values are found in the electrolyte volume closer to the metal surface than in the bulk. In addition, Mg0.6Ca shows a greater dynamics occurring at the surface location at which the vertical profiles were monitored, since the $\mathrm{pH}$ developing in the electrolyte volume adjacent to the surface greatly varies with the elapse of time. This behaviour is more acute at the beginning of the experiment, when electrolyte alkalization starts, but changes are still observed between the $\mathrm{pH}$ distributions measured after ca. 23.5 and 25.5 hours exposure to Ringer's solution. On the contrary, Mg0.9Ca shows a much more steady behaviour with the elapse of time, yet always exhibiting a continuous trend towards increased alkalization.

Area scans recorded over both alloys deliver similar observations. Figures 12 and 13 depict 2-D images of the $\mathrm{pH}$ distributions in the electrolyte for various exposure durations that were monitored by scanning the antimony microelectrode parallel to the surface of the alloys at a height of ca. 20-30 $\mu \mathrm{m}$. The images were selected among those recorded during the first few hours of immersion, and after ca. 24 hours immersion, respectively, and the scan dimensions were the same for both alloys in all the graphs. During the first few hours (see Figure 12), greater alkalization is always developed around the Mg0.9Ca, as result of the more intense cathodic activity occurring on this material, and the eventual redissolution of corrosion products according to reactions (2) and (3). Heterogeneous distributions are monitored over the two materials, though they present a greater variability for Mg0.9Ca, whose more active sites reach $\mathrm{pH}$ values near 11. The horizontal spike-lines observed in the $\mathrm{pH}$ distribution maps are likely to arise from local surface activation during scan acquisition. Such localized electrochemical activation would originate a transient potential change in the electrolyte that would affect the $\mathrm{pH}$ measurement by originating such spikes.

Regarding the $\mathrm{pH}$ distributions shown in Figure 13, the images recorded (cf. Figure 13A) do not match the same features observed in the $\mathrm{pH}$ distributions imaged at the longest exposures, which might be related to the solution refreshment operation performed after one night-exposure of the samples. The scans given in Figures 13B and C, that were recorder after a rather short stabilization period for the $\mathrm{pH}$ sensing probe, reproduce the same trends observed in earlier maps, though the corrosion process exhibits a more homogeneous distribution at the longest exposures.

\section{Conclusions}


The two MgCa alloys considered in this work, namely Mg-0.63Ca and Mg-0.89Ca, exhibited a single-phase $\alpha(\mathrm{Mg})$ solid solution. They are highly hydrophobous, closely resembling the wettability characteristics of pure magnesium.

Electrochemical characterization by electrochemical impedance spectroscopy of the alloys immersed in naturally aerated Ringer's solution at $37{ }^{\circ} \mathrm{C}$ delivered small impedance values (in the order of $10^{2} \Omega \mathrm{cm}^{2}$ ) from the medium to low frequency range, an indication of the vigorous dissolution process affecting the metallic materials. Precipitation of corrosion products occurred on the surface of the corroding MgCa alloys, as evidenced by the presence of an inductive region in the low frequency limit of the impedance spectra. EIS measurements showed that the corrosion process was under charge transfer control. Both MgCa alloys experienced non-uniform corrosion behaviour during the degradation process. This was evidenced through the potentiometric SECM analysis using antimony-based pHsensitive microelectrodes, which revealed the occurrence of non-uniform alkalization of the electrolyte over the two alloys. As result, $\mathrm{pH}$ variations in excess of 3 units between anodic and cathodic half-cell reactions were monitored. The more dynamic response given by the Mg0.63Ca sample under SECM characterization correlates well with its lower resistivity towards degradation as deduced from EIS measurements.

\section{Acknowledgments}

The authors are grateful for the HPT deformed MgCa alloys supplied by Professor Koichi Tsuchiya, and PhD students Fanqiang Meng and Baozhen Jiang from the Graduate School of Pure and Applied Sciences, University of Tsukuba, Japan. The work was supported by grants from the Romanian National Authority for Scientific Research (CNCS-UEFISCDI, project number PN-II-ID-PCE-2011-3-0218), and from the Spanish Ministry of Economy and Competitiveness and the European Regional Development Fund (Project No. CTQ2012-36787).

\section{References}

[1] M. Niinomi, T. Hattori, K. Morikawa, T. Kasuga, A. Suzuki, H. Fukui, S. Niwa, Development of low rigidity $\beta$-type titanium alloy for biomedical applications, Mater. Trans. 43 (2002) 2970-2977.

[2] M. Niinomi, Mechanical biocompatibilities of titanium alloys for biomedical applications, J. Mech. Behav. Biomed. Mater. 1 (2008) 30-42. 
[3] J. Nagels, M. Stkdijk, P.M. Rozing, Stress shielding and bone resorption in shoulder arthroplasty, J. Shoulder Elb. Surg. 12 (2003) 35-39.

[4] M.P. Staiger, A.M. Pietak, J. Huadmai, G. Dias, Magnesium and its alloys as orthopedic biomaterials: A review, Biomaterials 27 (2006) 1728-1734.

[5] R.C. Zeng, W. Dietzel, F. Witte, N. Hort, C. Blawert, Progress and challenge for magnesium alloys as biomaterials, Adv. Eng. Mater. 10 (2008) B3-B14.

[6] D. Xue, Y. Yun, M.J. Schulz, V. Shanov, Corrosion protection of biodegradable magnesium implants using anodization, Mater. Sci. Eng. C 31 (2011) 215-223.

[7] P. Rosemann, J. Schmidt, A. Heyn, Short and long term degradation behaviour of Mg-1Ca magnesium alloys and protective coatings based on plasma-chemical oxidation and biodegradable polymer coating in synthetic body fluid, Mater. Corros. 64 (2013) 714-722.

[8] D. Tie, F. Feyerabend, N. Hort, D. Hoeche, K.U. Kainer, R. Willumeit, W.D. Mueller, In vitro mechanical and corrosion properties of biodegradable Mg-Ag alloys, Mater. Corros. 65 (2014) 569-576.

[9] F.I. Wolf, A. Cittadini, Chemistry and biochemistry of magnesium, Mol. Aspects Med. 24 (2003) 3-9.

[10] N.E.L. Saris, Magnesium: an update of physiological, clinical and analytical aspects, Clin. Chim. Acta 294 (2000) 1-26.

[11] Z.J. Li, X.N. Gu, S.Q. Lou, Y.F. Zheng, The development of binary Mg-Ca alloys for use as biodegradable materials within bone, Biomaterials 29 (2008) 1329-1344.

[12] Y. Ding, C. Wen, P. Hodgson, Y. Li, Effects of alloying elements on the corrosion behavior and biocompatibility of biodegradable magnesium alloys: a review, J. Mater. Chem. B 2 (2014) 19121933.

[13] F. Witte, V. Kaese, H. Haferkamp, E. Switzer, A. Meyer-Lindenberg, C.J. Wirth, H. Windhagen, In vivo corrosion of four magnesium alloys and the associated bone response, Biomaterials 26 (2005) 3557-3563.

[14] F. Witte, The history of biodegradable magnesium implants: A review, Acta Biomater. 6 (2010) 1680-1692.

[15] B.A. Shaw, Corrosion resistance of magnesium alloys. In: ASM Handbook, Volume 13A Corrosion: Fundamentals, Testing, and Protection; ASM International, Materials Park, OH, 2003, pp. 692-696. 
[16] G. Song, Recent progress in corrosion and protection of magnesium alloys, Adv. Eng. Mater. 7 (2005) 563-586.

[17] M.B. Kannan, R.K.S. Raman, In vitro degradation and mechanical integrity of calcium containing magnesium alloys in modified-simulated body fluid, Biomaterials 29 (2008) 2306-2314.

[18] M. Salahshoor, Y. Guo, Biodegradable orthopaedic magnesium-calcium (MgCa) alloys, processing, and corrosion resistance, Materials 5 (2012) 135-155.

[19] M. Hollósi, Z.M. Shen, A. Perczel, G.D. Fasman, Stable intrachain and interchain complexes of neurofilament peptides: a putative link between $\mathrm{Al}^{3+}$ and Alzheimer disease, Proc. Natl. Acad. Sci. U.S.A. 91 (1994) 4902-4906.

[20] S. Rao, Y. Okazaki, T. Tateishi, T. Ushida, Y. Ito, Cytocompatibility of new Ti alloy without Al and V by evaluating the relative growth ratios of fibroblasts L929 and osteoblasts MC3T3-E1 cells, Materials Science and Engineering C 4 (1997) 311-314.

[21] J. Gröbner, D. Kervorkov, R. Schmidt-Fetzer, F.-W. Bach, H. Haferkamp, C. Jaschik, Materials design approaches and experiences; TMS, Warrendale, 2001.

[22] G. Song, Control of biodegradation of biocompatible magnesium alloys, Corros. Sci. 49 (2007) 1696-1701.

[23] F. Rosalbino, S. De Negri, A. Saccone, E. Angelini, S. Delfino, Bio-corrosion characterization of Mg-Zn-X (X = Ca, Mn, Si) alloys for biomedical applications, J. Mater. Sci. Mater. Med. 21 (2010) 1091-1098.

[24] X. Gu, Y. Zheng, S. Zhong, T. Xi, J. Wang, W. Wang, Corrosion of, and cellular responses to MgZn-Ca bulk metallic glasses, Biomaterials 31 (2010) 1093-1103.

[25] Y. Sun, B. Zhang, Y. Wang, L. Geng, X. Jiao, Preparation and characterization of a new biomedical Mg-Zn-Ca alloy, Mater. Des. 34 (2012) 58-64.

[26] A. Zakiyuddin, K. Lee, Effect of small addition of zinc and manganese to Mg-Ca based alloys on degradation behaviour in physiological media, J. Alloys Comp. 629 (2015) 274-283.

[27] Z. Li, X. Gu, S. Lou, Y. Zheng, The development of binary Mg-Ca alloys for use as biodegradable materials within bone, Biomaterials 29 (2008) 1329-1344.

[28] H.R.B. Rad, M.H. Idris, M.R.A. Kadir, S. Farahany, Microstructure analysis and corrosion behaviour of biodegradable Mg-Ca implant alloys, Mater. Des. 33 (2012) 88-97.

[29] X. Gu, Y. Zheng, Y. Cheng, S. Zhong, T. Xi, In vitro corrosion and biocompatibility of binary magnesium alloys, Biomaterials 30 (2009) 484-498. 
[30] Y.C. Li, C.E. Wen, D. Mushahari, R. Sravanthi, N. Harishankar, G. Pande, P. Hodgson, Mg-Zr-Sr alloys as biodegradable omplant materials, Acta Biomater. 8 (2012) 3177-3188.

[31] H.S. Brar, J. Wong, M.V. Manuel, Investigation of the mechanical and degradation properties of Mg-Sr and Mg-Zn-Sr alloys for use as potential biodegradable implant, J. Mech. Behav. Biomed. Mater. 7 (2012) 87-95.

[32] X.N. Gu, X.H. Xie, N. Li, Y.F. Zheng, L. Qin, In vitro and in vivo studies on a Mg-Sr bnary alloy system developed as a new kind of biodegradable metal, Acta Biomater. 8 (2012) 2360-2374.

[33] J.Z. Ilich, J.E. Kerstetter, Nutrition in bone health revisited: a story beyond calcium, J. Am. Coll. Nutr. 19 (2000) 715-737.

[34] J.J. Huang, Y.B. Ren, B.C. Zhang, K. Yang, Study on biocompatibility of magnesium and its alloys, Rare Metal Mat. Eng. 36 (2007) 1102-1105.

[35] N. Von Der Hoh, D. Bormann, A. Lucas, B. Denkena, C. Hackenbroich, A. Meyer-Lindenberg, Influence of different surface machining treatments of magnesium-based resorbable implants on the degradation behavior in rabbits, Adv. Eng. Mater. 11 (2007) B47-B54.

[36] N.T. Kirkland, N. Birbilks, J. Walker, T. Woodfield, G.J. Dias, M.P. Staiger, In-vitro dissolution of magnesium-calcium binary alloys: Clarifying the unique role of calcium additions in bioresorbable magnesium implant alloys, J. Biomed. Mater. Res. B 95 (2010) 91-100.

[37] Z. Shi, M. Liu, A. Atrens, Measurement of the corrosion rate of magnesium alloys using Tafel extrapolation, Corros. Sci. 52 (2010) 579-588.

[38] S. Thomas, N.V. Medhekar, G.S. Frankel, N. Birbilis, Corrosion mechanism and hydrogen evolution on Mg, Curr. Opin. Solid State Mater. Sci. 19 (2015) 85-94.

[39] S. Thomas, J. Izquierdo, N. Birbilis, R.M. Souto, Possibilities and limitations of scanning electrochemical microscopy of Mg and Mg alloys, Corrosion 71 (2015) 171-183.

[40] J. Izquierdo, L. Nagy, Á. Varga, J.J. Santana, G. Nagy, R.M. Souto, Spatially resolved measurement of electrochemical activity and $\mathrm{pH}$ distributions in corrosion processes by scanning electrochemical microscopy using antimony microelectrode tips, Electrochim. Acta 56 (2011) 8846-8850.

[41] S.E. Pust, D. Scharnweber, C. Nunes Kirchner, G. Wittstock, Heterogeneous distribution of reactivity on metallic biomaterials: Scanning probe microscopy studies of the biphasic Ti alloy Ti6Al4V, Adv. Mater. 19 (2007) 878-882. 
[42] S.E. Pust, D. Scharnweber, S. Baunack, G. Wittstock, Electron transfer kinetics at oxide films on metallic biomaterials: Scanning electrochemical microscopy studies of Ti6Al4V, J. Electrochem. Soc. 154 (2007) C508-C514.

[43] G. Ciurescu, J. Izquierdo, J.J. Santana, D. Mareci, D. Sutiman, S. González, R.M. Souto, Characterization of the localized surface chemical activity of Ti-Mo and Ti-Ta alloys for biomedical applications using scanning electrochemical microscopy, Int. J. Electrochem. Sci. 7 (2012) 7404-7424.

[44] J. Izquierdo, G. Bolat, D. Mareci, C. Munteanu, S. González, R.M. Souto, Electrochemical behaviour of ZrTi alloys in artificial physiological solution simulating in vitro inflammatory conditions, Appl. Surf. Sci. 313 (2014) 259-266.

[45] S. Iacoban, D. Mareci, G. Bolat, C. Munteanu, R.M. Souto, Multiscale electrochemical investigation of the corrosion resistance of various alloys used in dental prostheses, Met. Mater. Trans. B 46 (2015) 1011-1021.

[46] J. Izquierdo J, Nagy L, Bitter I, Souto RM, Nagy G. Potentiometric scanning electrochemical microscopy for the local characterization of the electrochemical behaviour of magnesium-based materials. Electrochim. Acta 87 (2013) 283-293.

[47] R.M. Souto, J. Izquierdo, J.J. Santana, A. Kiss, L. Nagy, G. Nagy, Progress in scanning electrochemical microscopy by coupling potentiometric and amperometric measurement modes. In: A. Méndez-Vilas (Ed.), Current Microscopy Contributions to Advances in Science and Technology, Formatex Research Center, Badajoz, 2012, pp. 1407-1415.

[48] ASTM G1-90: Standard practice for preparing, cleaning, and evaluating corrosion test specimens, American Society for Testing \& Materials, West Conshohocken, PA, 1999.

[49] J. Cheng, B. Liu, Y.H. Wu, Y.F. Zheng, Comparative in vitro study on pure metals (Fe, Mn, Mg, Zn and W) as biodegradable metals, J. Mater. Sci. Technol. 29 (2013) 619-627.

[50] J. Chen, J.Q. Wang, E.H. Han, W. Ke, ESEM observation of the process of hydrogen generation around the micro-droplets forming on AZ91 magnesium alloy, Electrochem. Commun. 10 (2008) 577-581.

[51] X. Liu, D. Shan, Y. Song, R. Chen, E. Han, Influences of the quantity of $\mathrm{Mg}_{2} \mathrm{Sn}$ phase on the corrosion behaviour of Mg-7Sn magnesium alloy, Electrochim. Acta 56 (2011) 2582-2590. 
[52] H. Wang, Y. Estrin, H.M. Fu, G.L. Song, Z. Zuberova, The effect of pre-processing and grain structure on the bio-corrosion and fatigue resistance of magnesium alloy AZ31, Adv. Eng. Mater. 9 (2007) 967-972.

Table 1. Electrochemical parameters obtained from EIS spectra using the selected EC for both MgCa alloys after different immersion time in Ringer's solution, at $37^{\circ} \mathrm{C}$

\begin{tabular}{|c|c|c|c|c|}
\hline $\begin{array}{c}\text { Immersion } \\
\text { time }\end{array}$ & $\begin{array}{c}10^{4} Q_{1} / \\
\mathrm{S} \mathrm{cm}^{-2} \mathrm{~s}^{n}\end{array}$ & $n_{1}$ & $\begin{array}{c}R_{1} / \\
\Omega \mathrm{cm}^{2}\end{array}$ & $\begin{array}{c}L / \\
\mathrm{mH} \mathrm{cm}\end{array}$ \\
\hline \multicolumn{5}{|c|}{ Mg0.6Ca alloy } \\
\hline 1 hour & 4.5 & 0.82 & 467 & 6.9 \\
\hline 1 day & 5.8 & 0.81 & 228 & 4.6 \\
\hline 1 week & 7.1 & 0.80 & 98 & 1.8 \\
\hline \multicolumn{7}{|c|}{$\mathbf{M g 0 . 9 C a}$ alloy } \\
\hline 1 hour & 3.9 & 0.84 & 785 & 16.1 \\
\hline 1 day & 4.1 & 0.83 & 679 & 12.4 \\
\hline 1 week & 6.7 & 0.81 & 367 & 6.3 \\
\hline
\end{tabular}



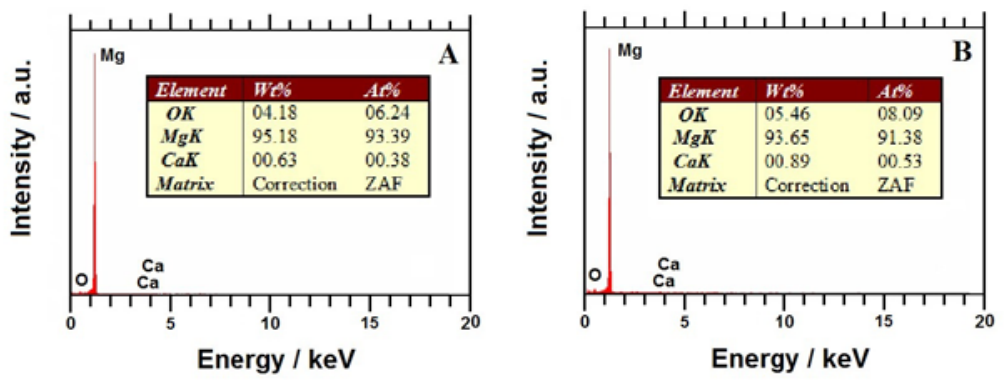

Figure 1. EDX data determined for as-cast: (A) Mg0.6Ca alloy, and (B) Mg0.9Ca alloy.

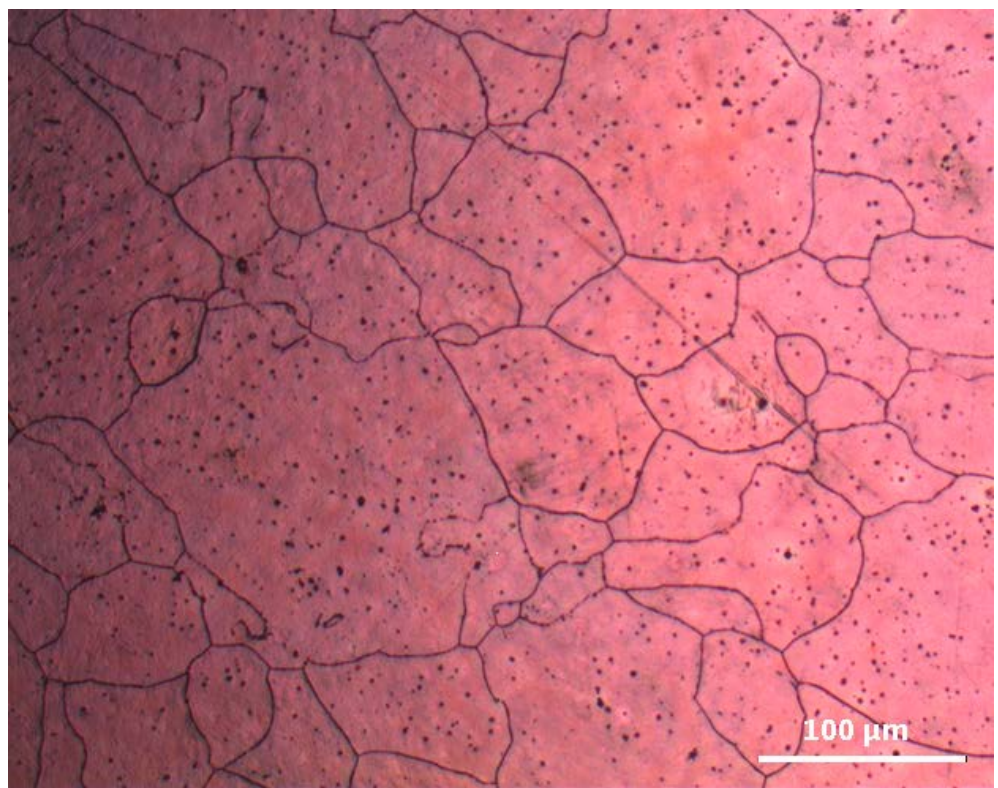

Figure 2. Microstructure of Mg0.9Ca alloy analyzed by optical microscopy. 


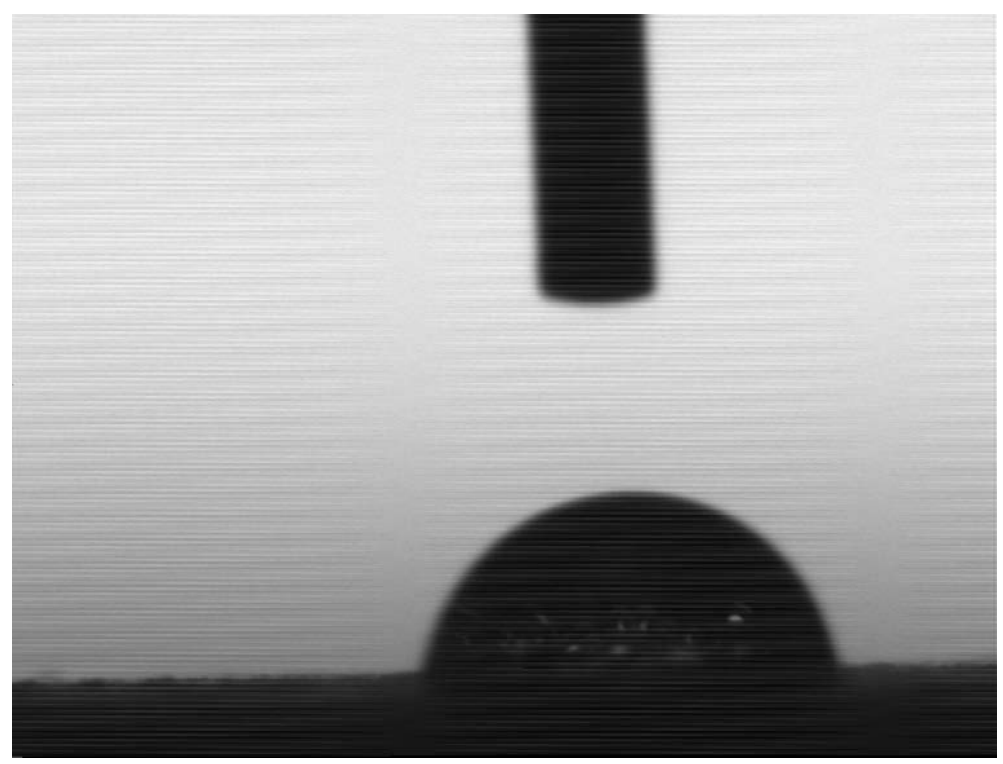

Figure 3. Shape of the liquid drop applied on the surface of the Mg0.9Ca alloy retrieved from naturallyaerated Ringer’s solution after 1 week.
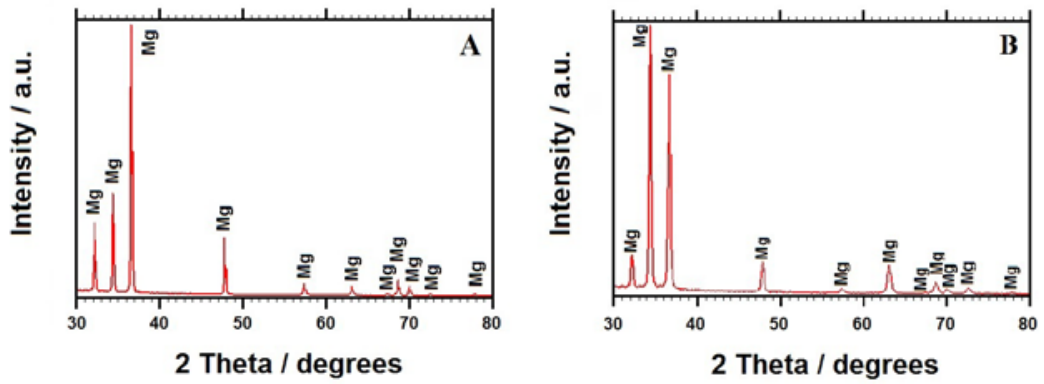

Figure 4. X-ray diffractometry patterns measured for (A) Mg0.6Ca and (B) Mg0.9Ca alloys. 

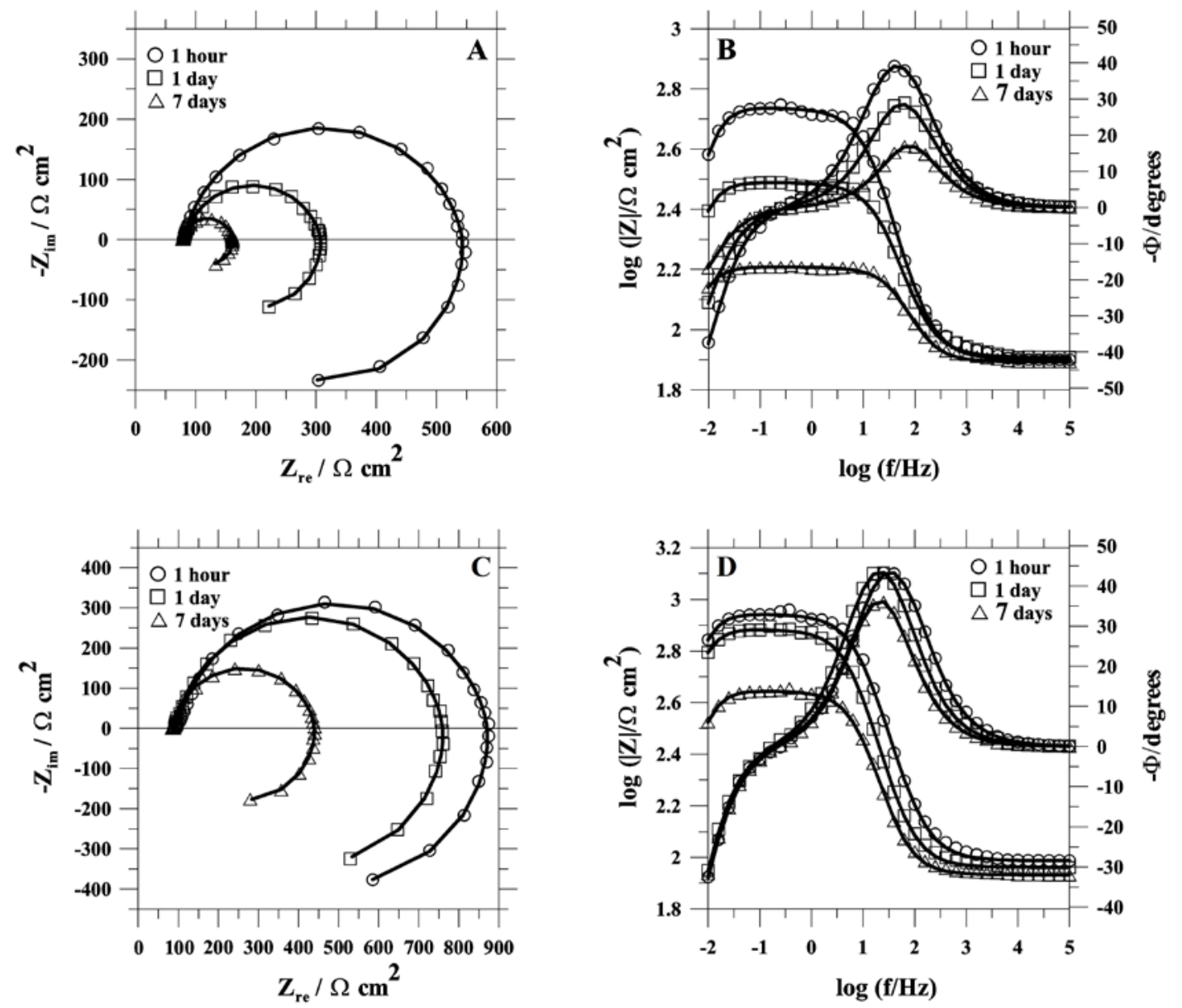

Figure 5. Nyquist and Bode diagrams for (A,B) Mg0.6Ca and (C,D) Mg0.9Ca alloys after different immersion times in naturally aerated Ringer's solution at $37^{\circ} \mathrm{C}$, measured at the open circuit potential.

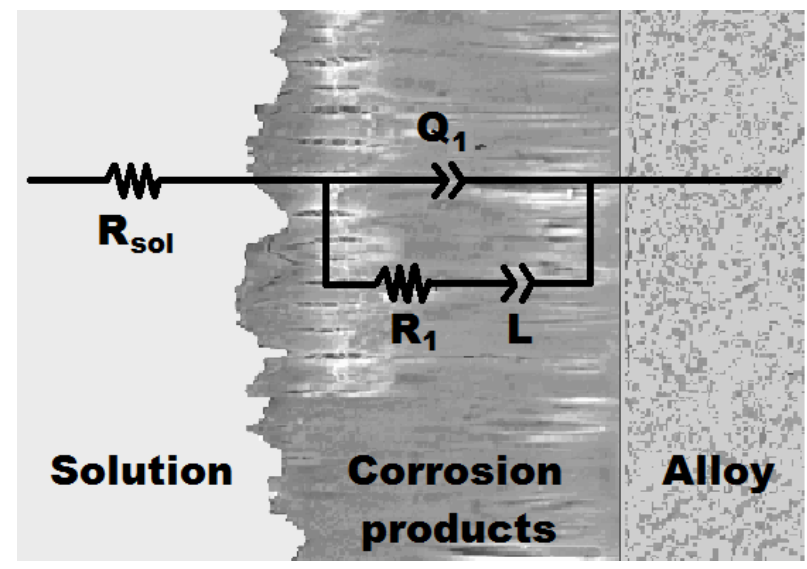

Figure 6. Equivalent circuit (EC) used to analyze the impedance spectra. 


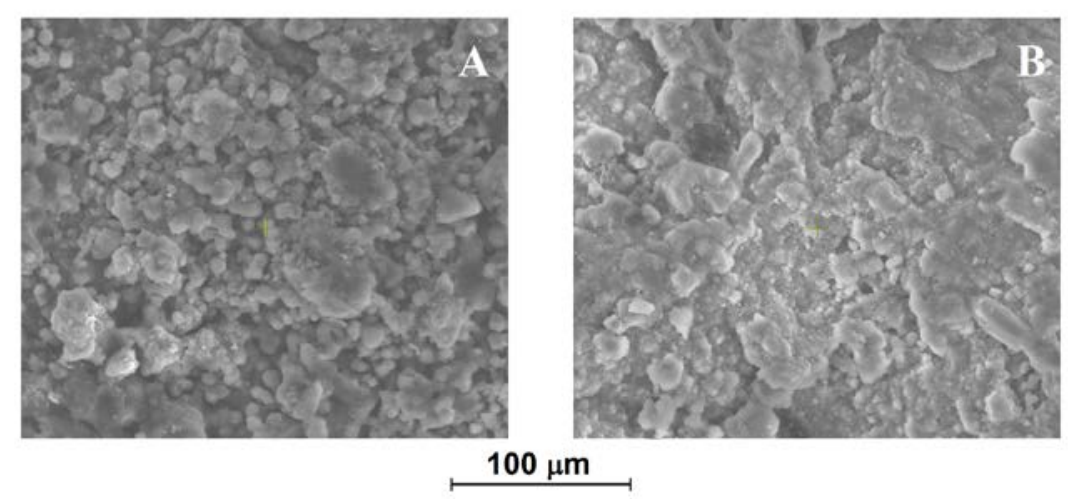

Figure 7. SEM images showing the surface of (A) Mg0.6Ca and (B) Mg0.9Ca alloys retrieved from naturally aerated Ringer's solution at $37^{\circ} \mathrm{C}$ after 1 week.
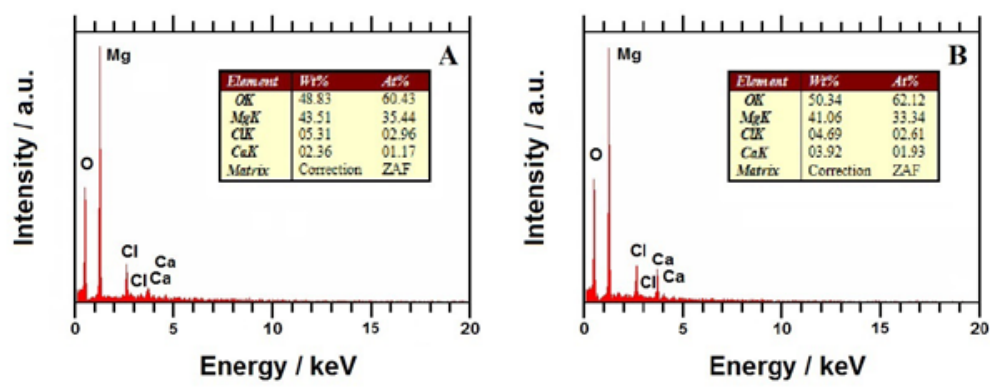

Figure 8. EDX spectra from corroded (A) Mg0.6Ca and (B) Mg0.9Ca alloys retrieved from naturally aerated Ringer's solution at $37^{\circ} \mathrm{C}$ after 1 week.
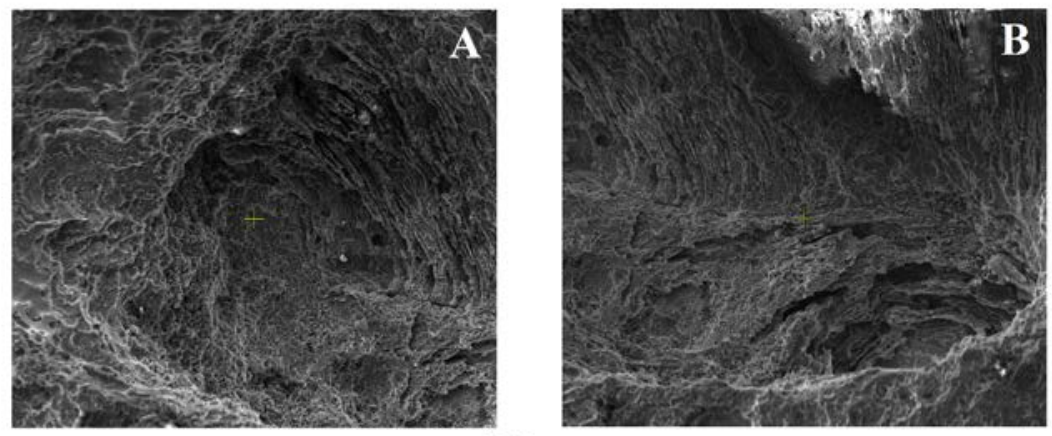

$500 \mu \mathrm{m}$

Figure 9. SEM images showing the corroded underlying surface of (A) Mg0.6Ca and (B) Mg0.9Ca alloys after removal of the corrosion products deposited during 1 week immersion in naturally aerated Ringer's solution at $37^{\circ} \mathrm{C}$. 

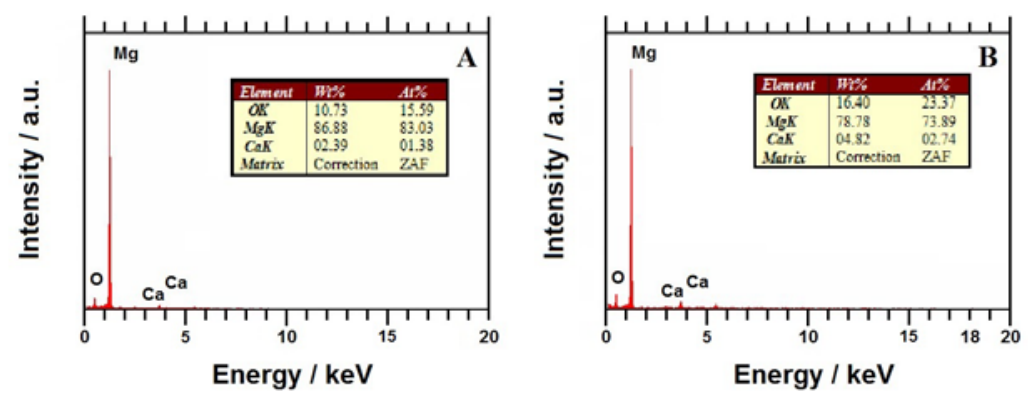

Figure 10. EDX spectra of the corroded underlying surface of (A) Mg0.6Ca and (B) Mg0.9Ca alloys after removal of the corrosion products deposited during 1 week immersion in naturally aerated Ringer's solution at $37^{\circ} \mathrm{C}$.
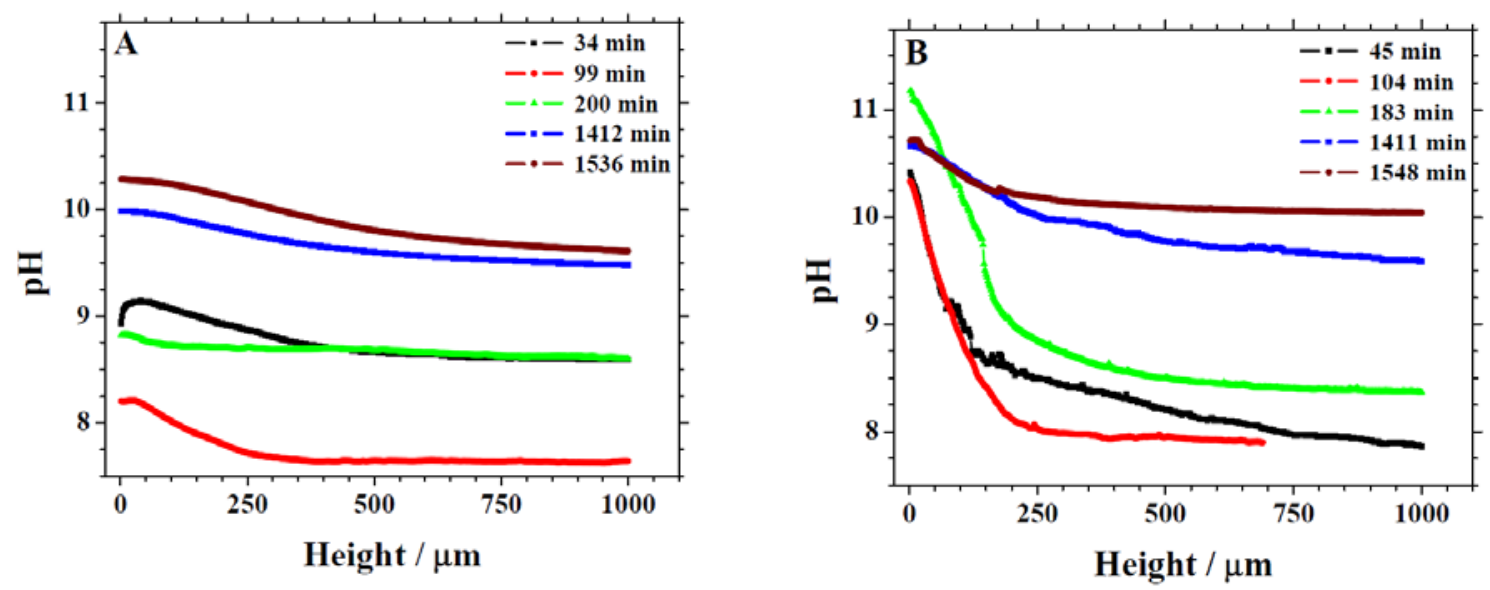

Figure 11. Evolution of $\mathrm{pH}$ distribution above (A) Mg0.6Ca and (B) Mg0.9Ca alloys in naturally aerated Ringer's solution at $37^{\circ} \mathrm{C}$ for the immersion times given in the graphs. 




A

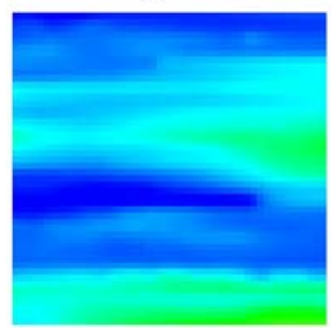

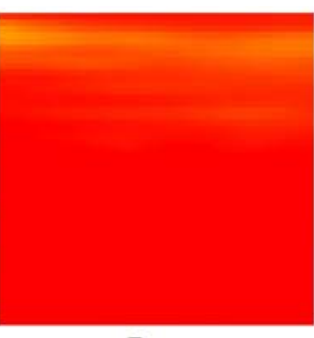

B

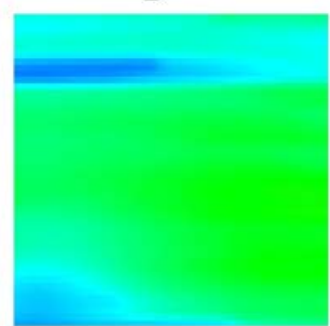

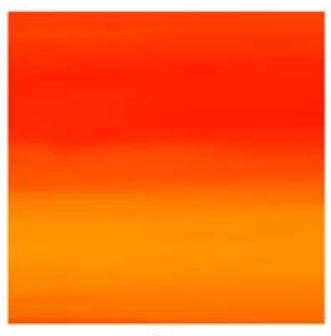

C

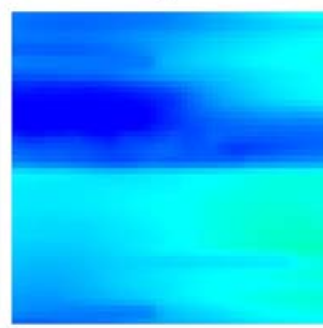

$\mathrm{pH}$
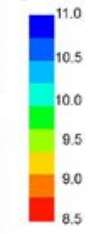

Figure 12. $\mathrm{pH}$ distribution in a plane parallel to the surface of (above) Mg0.6Ca and (below) Mg0.9Ca alloys after (A) 0.5, (B) 2.5 and (C) 3 hours immersion in naturally aerated Ringer's solution at $37^{\circ} \mathrm{C}$. Scan dimensions: $250 \mu \mathrm{m} \times 250 \mu \mathrm{m}$.

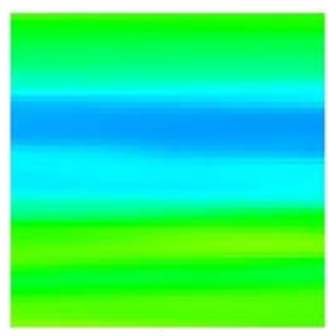

A

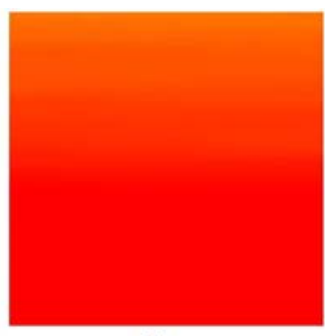

B

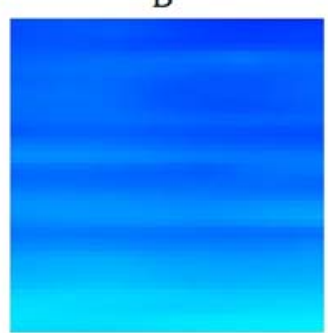

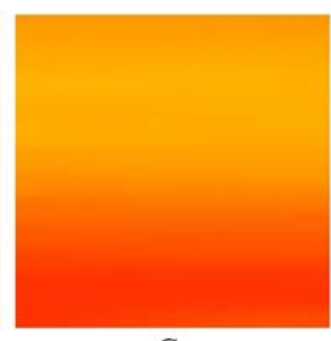

C



Figure 13. $\mathrm{pH}$ distribution in a plane parallel to the surface of (above) Mg0.6Ca and (below) Mg0.9Ca alloys after (A) 22.5, (B) 23 and (C) 24 hours immersion in naturally aerated Ringer’s solution at $37^{\circ} \mathrm{C}$. Scan dimensions: $250 \mu \mathrm{m} \times 250 \mu \mathrm{m}$. 